organisations. This multi-component approach included: the development of dementia friendly hospice building; an enhanced hospice/dementia specialist team; a day hospice service for persons with dementia and their carers which provided opportunities for nurse specialist assessment, creative therapies to improve cognition and promote well-being for the person, and complementary therapy; development of education and learning through certificate programme in holistic dementia care.

HEDP has fostered the development of strong partnerships between participating organisations and has facilitated the cross-fertilisation of expertise. External practitioners and hospice staff have benefitted from integrating learning from mental health, palliative care and other areas of practice.

Aim To profile findings from the evaluation of the HEDP in terms of uptake, the experiences of carers, health and social care professionals, service commissioners and policy makers, and to highlight recommendations for service development, education and practice.

Methods Multiple methods: data collection including documentary record analysis, interviews, focus groups and analysis of reflective diary accounts. Ethical approval was secured. The evaluation was undertaken by researchers independent of the hospice. Descriptive statistical analysis with chart data and thematic analysis applied to qualitative data.

Results/conclusion Core findings from the evaluation process highlighted four main themes: (i)The impact of dementia, (ii) Value of service, (iii) Information and learning needs and (iv) Working in partnership, and has developed key recommendations for teams, commissioners and policy makers considering developing integrated approaches between palliative and dementia care.

\section{0-12 COPING WITH GRIEF: IDENTIFYING CORE OUTCOMES FOR EVALUATING BEREAVEMENT SUPPORT IN PALLIATIVE CARE}

${ }^{1}$ Emily Harrop, ${ }^{1}$ Hannah Scott, ${ }^{1}$ Kathy Seddon, ${ }^{1} \mathrm{Jim}$ Fitzgibbon, ${ }^{2}$ Fiona Morgan, ${ }^{3}$ Sara Pickett, ${ }^{1}$ Anthony Byrne, ${ }^{4}$ Stephanie Sivell, ${ }^{4}$ Mirella Longo. ${ }^{1}$ Marie Curie Research Centre, Cardiff University, Cardiff, UK; ${ }^{2}$ Specialist Unit for Review Evidence, Cardiff University, Cardiff, UK; ${ }^{3}$ Swansea Centre for Health Economics, Swansea University, Swansea, UK; ${ }^{4}$ Marie Curie Research Centre, Cardiff, UK

\subsection{6/bmispcare-2018-hospiceabs.12}

Background Bereavement support is considered an essential part of hospice provision. However, the research evidence is limited by a lack of consistency in the outcomes used to evaluate services, which in turn makes it difficult to compare approaches and draw conclusions on 'what works best'. Core Outcome Sets (COS) represent the 'minimum that should be measured and reported' in research into specific conditions or services [www.comet-initiative.org]. This project aimed to develop a COS for evaluating bereavement support in palliative care for adults who have lost adults through terminal illness.

Methodology The research began with a systematic review of quantitative and qualitative literature to identify a list of outcomes relevant to bereavement support. At a subsequent consensus day 21 stakeholders (including service users) discussed what they considered to be the most important outcomes and compared these to and critiqued the lists generated from the review. These lists and discussions informed a two round DELPHI survey $(n=238)$ designed to reach consensus on which outcomes/outcome dimensions should be included in the Core Outcome Set. During a final consensus day participants ranked the relative importance of the items which reached consensus in the survey.

Results Results suggest that the two most important outcomes are 'Ability to cope' and 'Quality of life and mental wellbeing'. A number of core dimensions to explore when assessing these outcomes have also been identified. Examples include; 'Feelings of loneliness and emptiness'; 'Relationships with friends and family'; 'Ability to find balance and channel grief'; 'Ability to perform daily tasks' and 'Sense of meaning and purpose in life'.

Conclusion Available validated tools have been reviewed for their relevance to these outcomes. No tools have been identified which cover all core domains, although some have better 'fit' than others. Further work is being planned to adapt or develop measures as necessary.

\section{0-13 HOW DARE WE?! - DISCHARGING PALLIATIVE PATIENTS FROM HOSPICE SERVICES}

Lara Cowley. St Wilfrid's Hospice, Eastbourne, East Sussex

\subsection{6/bmjspcare-2018-hospiceabs. 13}

Background With the steady rise of annual deaths, increased patient complexity, and ongoing financial constraints there is a threat to how hospices can continue to deliver the excellent care they are renowned for. As hospices adopt working models to cope with these ever increasing challenges it is imperative that services are delivered in a timely manner. To best achieve this, hospices need to examine their patterns of patient input and the level of care that is delivered throughout a patient's journey. A way of balancing demand would involve hospices being more proactive in discharging patients when stable. With the development of the Rehabilitative Palliative Care model and the suite of OACC measures, hospices are now in a position to utilise this approach.

Aim To explore how to facilitate discharging patients from hospice services appropriately without impacting on care.

Methods Systematic literature review and qualitative study using a patient focus group.

Results Evidence shows that facilitating appropriate access to hospice services requires understanding of staff and patient behaviours. Findings show hospice staff often provide a 'total care' approach leading to increased patient dependency and are uncomfortable with the notion of discharging patients. Although research is limited, early findings suggest patients develop a complex bond with their hospice and view it as a place that fills the void and gives meaning to their life. This creates practical and ethical barriers to discharging patients, especially when there is unlikely another service that matches hospice care.

Conclusions Ongoing research is needed but there is the recognition that discharging hospice patients is a sensitive topic and raises concerns how this can be managed and perceived. However, there are strategies that can be implemented to aid this and given the current and ongoing challenges hospices face is this a conversation we can no longer avoid? 